\title{
To investigate the effect of Mobile IP on the performance of VANET through realistic channel conditions.
}

Komal Sharma, Jyoteesh Malhotra

Deparment of ECE, Guru Nanak Dev University R.C Jalandhar

Komal.10ece@gmail.com

Department of ECE , Guru Nanak Dev University R.C Jalandhar Jyoteesh@gmail.com

\section{ABSTRACT}

Vehicular Ad hoc Network (VANET) is a specialized Ad hoc Network, which provides safety and comfort for passengers [1]. Due to the specific characteristic of VANET like high mobility and large scale node population [1], providing Quality of Service (QoS) in this type of wireless network is a challenging issue. As a result, we combine Mobile IP and VANET to improve QoS in terms of packet loss and throughput for traffic safety and entertainment applications. Comparative performance evaluation is done in terms of QOS parameters to show the network performance using different traffic types and by varying speed of the vehicles under urban scenario.

\section{Indexing terms/Keywords}

VANET, ITS, V2V, V2I, RSU and OBU

\section{Council for Innovative Research}

Peer Review Research Publishing System

\section{Journal: INTERNATIONAL JOURNAL OF COMPUTERS \& TECHNOLOGY}

Vol.14, No.7

www.ijctonline.com, editorijctonline@gmail.com 


\section{Introduction}

VANET is a type of Ad-hoc networks, which provides wireless communication between V2V and V2I. To connect all the moving vehicles to the internet is the most important requirement for the operation of VANET. As, VANET is an wireless network while internet is an wired network. Thus for combining these heterogeneous networks, Mobile IP is used to support IP mobility of mobile nodes in wireless network with infrastructure. Mobile IP is an open standard, defined by the Internet Engineering Task Force (IETF) RFC 2002[2], that allows users to keep the same IP address, stay connected, and maintain ongoing applications while roaming between IP networks. The combination of VANET and Mobile IP concept provides long range communication and the location of the vehicle can be tracked very easily. It also provide the information like driver's identity, location of keys and also provide authentication services for the security puposes. When the vehicle moves away from the range of current serving RSU, to the region of another RSU the signal strength of previous RSU goes on decreasing and the signal strength of new RSU goes on increasing, Thus handover is required, to avoid packet drop or delay in packet delivering the handoff should be a soft handoff i.e the connection to new RSU should be established before the connection termination to the previous RSU.

The concept of Mobile IP in VANET enables the vehicle to access the RSU without losing connection. As the vehicle is highly mobile and dynamic in nature, thus handover is required between the vehicle and RSU[3], whenever the vehicle move from the range of existing RSU to the new RSU. As the vehicle is moving from one place to another place, it must requires same IP address so that vehicle can be tracked easily and exiting connection can be maintained. To provide better QOS parameters the vehicle should be in the coverage range of RSU for the maximum time period, time delay required for sending and receiving the data packets should be less, throughput rate should be as high as possible and packet drop rate should be minimum.

The remainder of paper is organized as follows: Section II provides a brief study about Mobile IP. Section III indicates system model and Section IV presents analysis of results and finally section $V$ concludes the paper.

\section{Mobile IP}

In Mobile IP routing of the packets is based on stationary IP addresses similar to a postal letter are delivered to the fixed address on the envelope. Thus, IP address of a device is always fixed and we can track the location of any device from its IP address.

Whenever mobile device (vehicle) roams away from the range of its home network, it becomes difficult to maintain the ongoing sessions. Thus, the concept of Mobile IP was created to allow users to keep the same IP address, stay connected and maintain ongoing communication without delay[4]. Also, certain network services, such as software licenses and access privileges, location tracking are based on IP addresses.

\section{i) Components of a Mobile IP Network}

Mobile IP consists of three major components.

\section{- Mobile Node}

- Home Agent

- Foreign Agent

The concept of Mobile IP can be analysed, as shown in Figure 1:

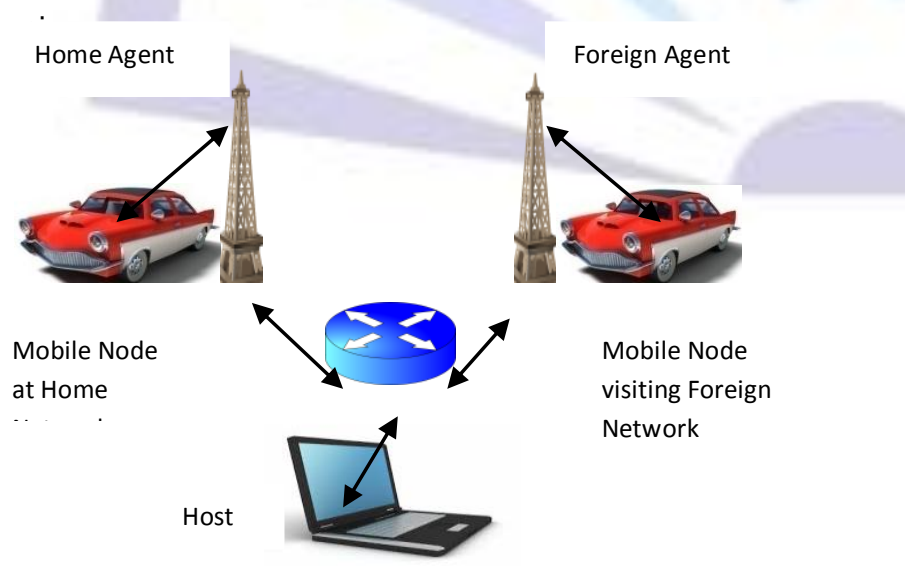

Figure 1 Mobile IP Components and their relationship in VANET.

The Mobile Node is a device such as car, bus, truck or any vehicle which consist of OBU (on board circuit unit) whose software enables V2V \& V2I network communication. 
The Home Agent can be defined as RSU in the home network which serves as the anchor for[5] communication with the Mobile Node (vehicle). A tunnel is established between the Home Agent and Foreign Agent and home agent tunnels the packets to the mobile vehicle in the foreign network.

The Foreign Agent can be defined as an access point (RSU) that is responsible for delivering packets from home network to vehicle when it roams to a foreign network.

\section{ii) How Mobile IP Works}

This section explains the working of Mobile IP. The process of Mobile IP has three main phases, which are discussed in the following sections.

- $\quad$ Agent-Discovery

In this phase Mobile Node discovers its Foreign and Home Agents.

- Registration

During registration process Mobile Node registers its current location with the Foreign Agent and Home Agent.

- Tunneling

A reciprocal tunnel is set up by the Home Agent to the care-of address (current location of the vehicle on the foreign network) to route packets to the vehicle as it roams.

As, shown in the figure1, the host maintains all the information about the vehicle as it moves from the home agent to the foreign agent, it updates vehicle's current location and RSU's (agents) provides the information about the if there is any construction work ongoing on the road, traffic accident occurred, the turning point of the road, the status of the road weather it is congested or not, So that time can be saved by choosing some another way. It also provides all the necessary information like when to overtake the car in front of you specially in case of single road with incoming and outgoing traffic. It provides the online map about the current city in which the vehicle has entered like the current available space for parking, nearest petrol pumps with rates[9]. It also saves our time by automatically switches the red light to green light when the road is empty.

\section{System Model}

In this paper city scenario with Mobile IP configuration has been considered. For this purpose Okumara model is considered because it is best suited model for the urban scenario and the NCTUns-6.0 simulation tool is considered for result evaluation.

\section{i) Okumara Model}

The Okumura model is a subtype of empirical channel models used in VANET. This model is designed from the empirical data collected in detailed propagation tests over various situations of an irregular terrain and environmental clutter. This is one of the widely used models in urban areas and can be expressed as [7]:

$$
L_{50}=L_{F}+A_{m u}(f, d)-G\left(h_{t e}\right)-G\left(h_{r e}\right)-G_{A R E A}
$$

Where,

$\mathrm{L}_{50}$ : median value of propagation path loss,

$\mathrm{L}_{\mathrm{F}}$ free space propagation path loss,

$A_{m u}$ median attenuation in the medium,

$f$ : free space at frequency,

d: distance between the RSU and OBU ,

$\mathrm{G}\left(\mathrm{h}_{\mathrm{te}}\right)$ : Gain factor of RSU,

$\mathrm{G}\left(\mathrm{h}_{\mathrm{re}}\right)$ : Gain factor of OBU,

$h_{\mathrm{te}} \& h_{\mathrm{re}}$ : effective heights of base station and mobile antennas (in meters),

$\mathrm{G}_{\text {AREA: }}$ gain generated by the environment at which the system is operating and it can be obtained from the empirical curves.

\section{ii) Simulation Scenario}

The scenario is drawn with "draw topology" feature of NCTUns-6.0 simulator. Then using "edit topology" feature required simulation parameters are adjusted and then using "Run" feature results are evaluated[10]. 


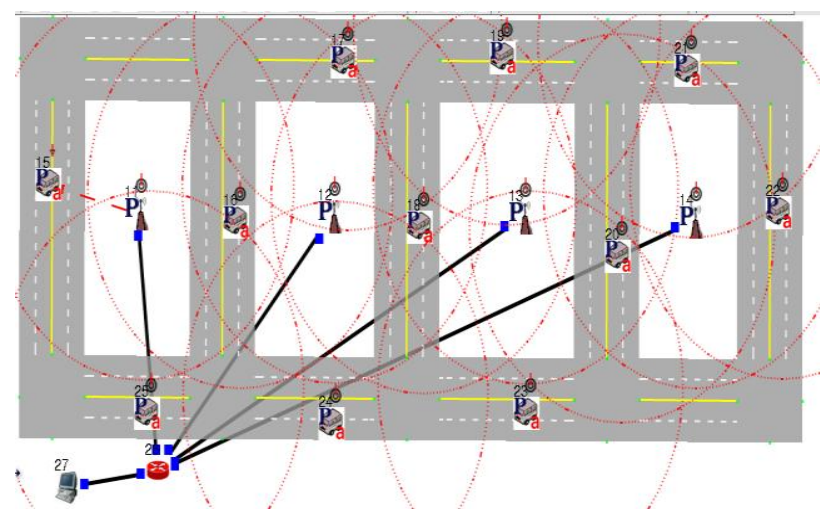

Figure2: Vehicle in Home Network.

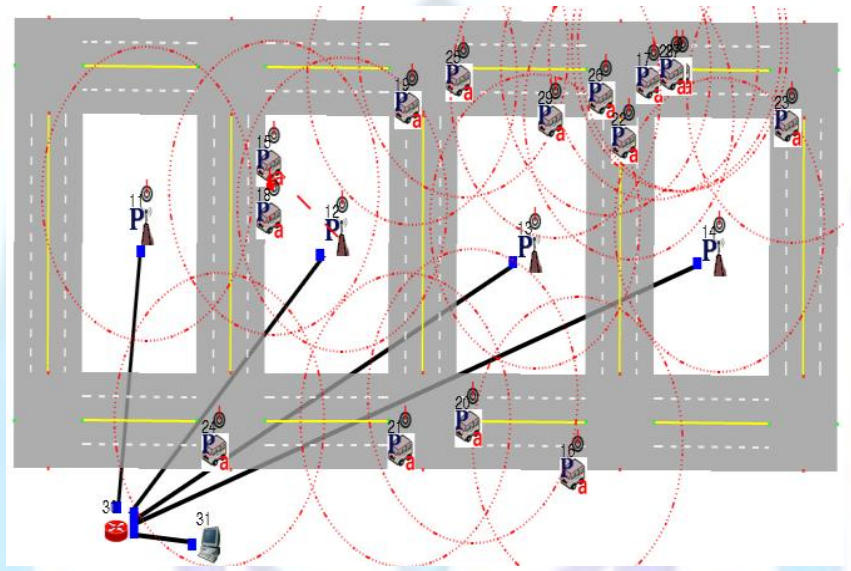

Figure3: Vehicle in Foreign Network.

\section{ii) Simulation Parameters}

After the simulation scenario is drawn, certain simulation parameters are required to be selected or adjusted according to the requirement. The list of simulation parameter selection is given in ab table below:

\section{Table1: Simulation Parameters:}

\begin{tabular}{|l|l|}
\hline Visualization & NCTUns \\
\hline MAC & $802.11 \mathrm{p}$ \\
\hline $\begin{array}{l}\text { Ad-hoc Routing } \\
\text { Protocol }\end{array}$ & AODV \\
\hline Mobility Model & Manhattan \\
\hline No. of Vehicles & 15 \\
\hline $\begin{array}{l}\text { No. of Base } \\
\text { Stations(RSU) }\end{array}$ & 4 \\
\hline Speed of Vehicles & $36 \mathrm{~km} / \mathrm{hr} ; 72 \mathrm{~km} / \mathrm{hr}$ \\
\hline Transport Protocol & UDP \\
\hline $\begin{array}{l}\text { Empirical Channel } \\
\text { Model }\end{array}$ & Okumara \\
\hline $\begin{array}{l}\text { Traffic type } \\
\text { Antenna Height }\end{array}$ & stg, stcp \\
\hline Target Peak data rate & $6 \mathrm{Mbps}$ \\
\hline
\end{tabular}


In our study performance of various routing protocols can be compared based on the three parameters namely Throughput, Packet Drop and collision rate as follows.

\section{RESULTS \& DISCUSSIONS}

After running the above scenario.The results are evaluated as given below:

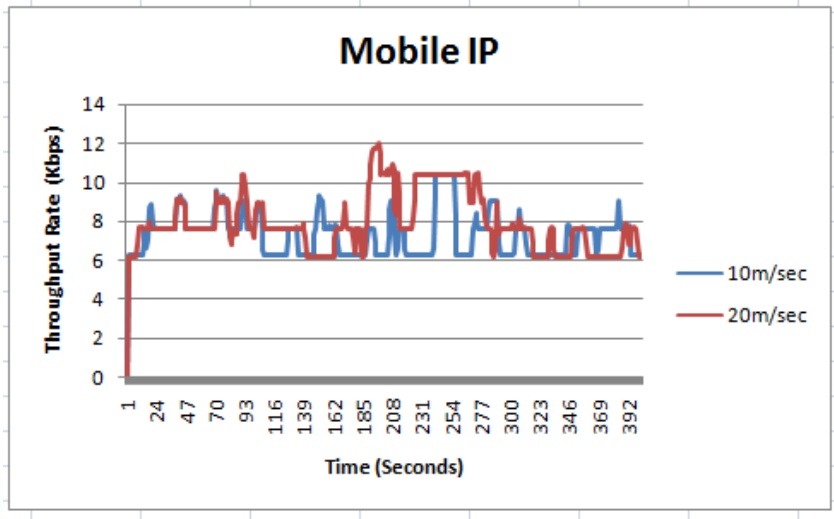

Figure3: Throughput Rate using 'stg' Traffic type.

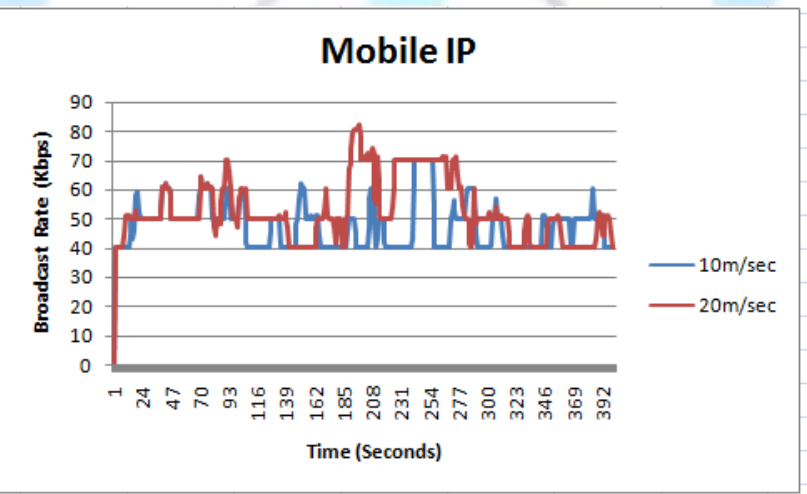

Figure4: Broadcast Rate using 'stg' Traffic type.

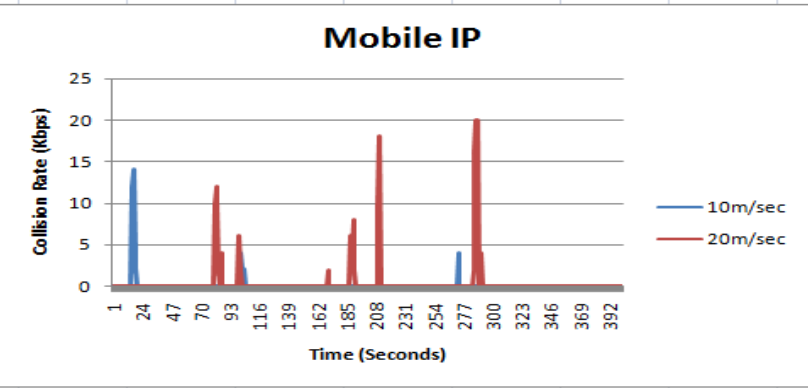

Figure5:Collision Rate using 'stg' Traffic type.

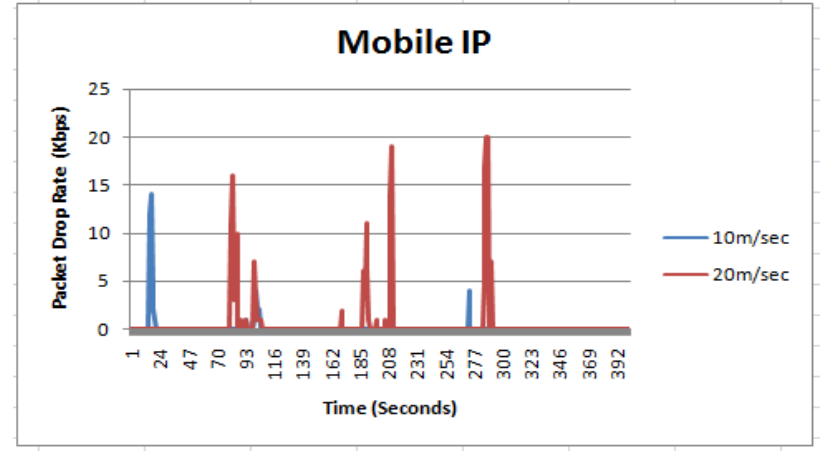

Figure6: Packet Drop Rate using 'stg' Traffic type. 


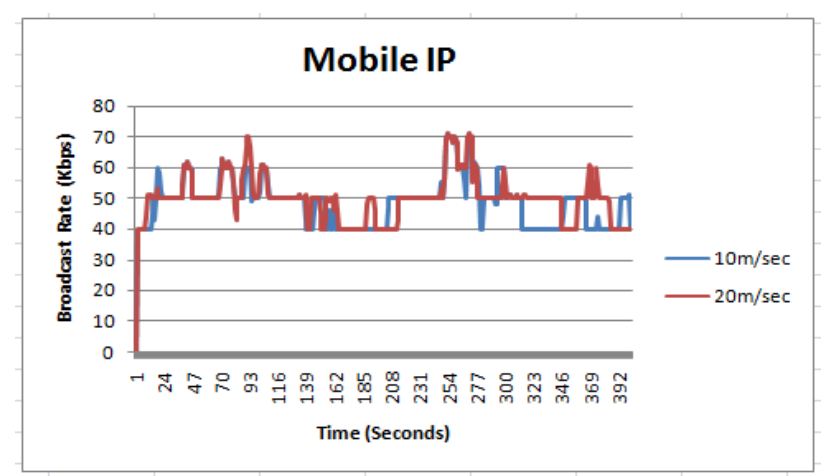

Figure7: Broadcast Rate using 'stcp' Traffic type.

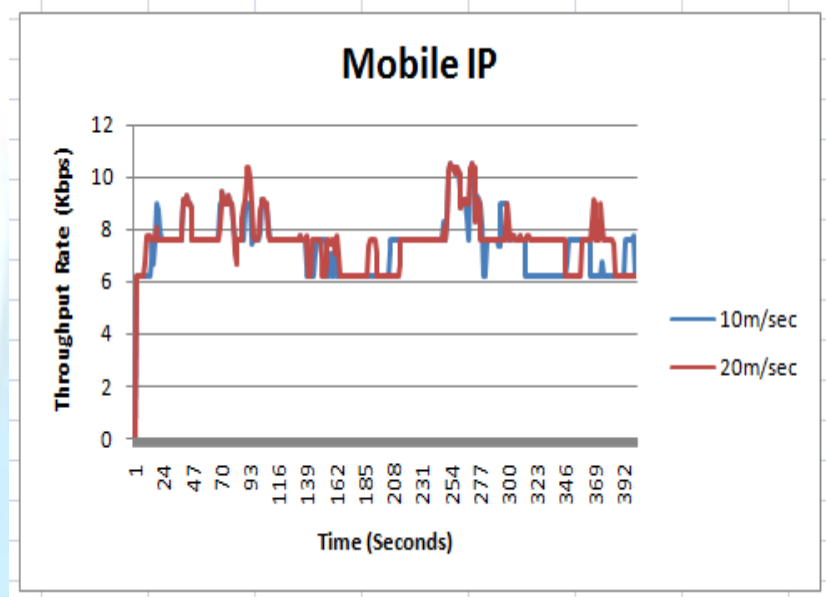

Figure8: Throughput Rate using 'stcp' Traffic type.

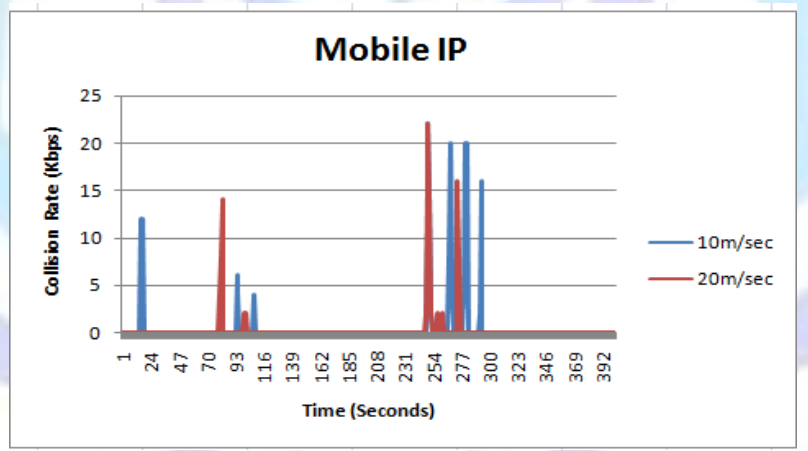

Figure9: Collision Rate using 'stcp' Traffic type.

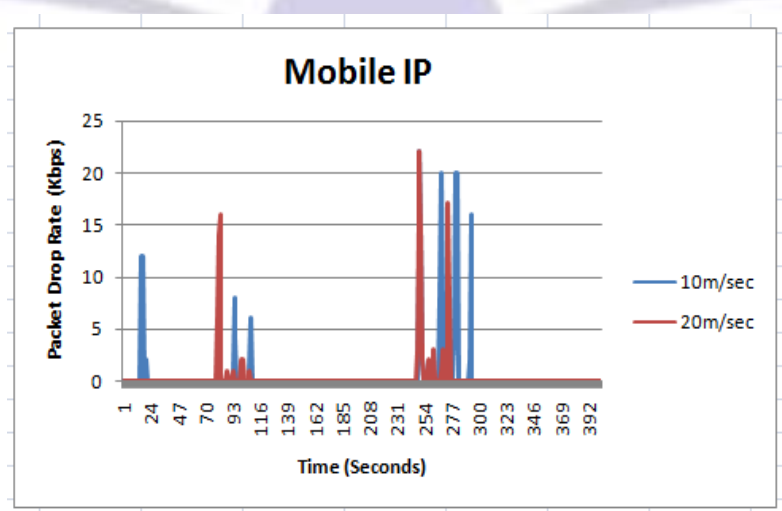

Figure10: Packet Drop Rate using 'stcp' Traffic type.

The simulation results are taken in terms of various quality of service parameters such as throughout rate, collision rate, packet drop rate and broadcast rate at different speeds and using different traffic types i.e stg/rtg \& stcp/rtcp. STG can be 
defined as State Transition Graph, it first calculates the traffic flow and then saved to the output file. It do not provide control function while STCP is a generic, scalable and reliable transport layer protocol where a majority of the functionalities are implemented at the base station. STCP offers controlled variable reliability, congestion detection and avoidance, and supports multiple applications in the same network. The results are summarized in a table given below for better analyses.

Table2: Comparison of performance parameters.

\begin{tabular}{|c|c|c|c|c|}
\hline TRAFFIC & \multicolumn{2}{|l|}{ STCP } & \multicolumn{2}{|l|}{ STG } \\
\hline PARAMETERS & $\begin{array}{l}\text { AT } \\
\text { SPEED } \\
\text { OF } \\
10 \mathrm{~m} / \mathrm{s} \\
\text { or } \\
32 \mathrm{~km} / \mathrm{h}\end{array}$ & $\begin{array}{l}\text { AT } \\
\text { SPEED } \\
\text { OF } \\
20 \mathrm{~m} / \mathrm{s} \\
\text { or } \\
72 \mathrm{~km} / \mathrm{h}\end{array}$ & $\begin{array}{l}\text { AT } \\
\text { SPEED } \\
\text { OF } \\
10 \mathrm{~m} / \mathrm{s} \\
\text { or } \\
32 \mathrm{~km} / \mathrm{h}\end{array}$ & $\begin{array}{l}\text { AT } \\
\text { SPEED } \\
\text { OF } \\
20 \mathrm{~m} / \mathrm{s} \\
\text { or } \\
72 \mathrm{~km} / \mathrm{h}\end{array}$ \\
\hline $\begin{array}{l}\text { Throughput } \\
\text { Rate(Kbps) }\end{array}$ & 7.41 & 7.58 & 7.27 & 7.94 \\
\hline $\begin{array}{l}\text { Packet Drop } \\
\text { Rate(Kbps) }\end{array}$ & 0.55 & 0.34 & 0.53 & 0.12 \\
\hline $\begin{array}{l}\text { Collision } \\
\text { Rate(Kbps) }\end{array}$ & 0.52 & 0.28 & 0.40 & 0.11 \\
\hline $\begin{array}{l}\text { Broadcast } \\
\text { Rate(Kbps) }\end{array}$ & 48.53 & 49.79 & 47.60 & 52.50 \\
\hline
\end{tabular}

From the table above it can be evaluated that the throughput rate and broadcast rate increases with the speed of the vehicle because it provides quick handoff as the movement of vehicle from the coverage area of one RSU to another RSU is very fast. Thus, delay is minimum which lead to increase in throughput rate and broadcast rate. If we compare the traffic types we can find that the STCP provides better performance over STG because of its features like congestion detection and control applications which are not present in STG. Thus, the throughput rate is more in case of STCP while packet drop rate and collision rate is less.

\section{CONCLUSION}

In this paper performance parameters are compared in urban scenario with Mobile IP application on the basis of the speed of the vehicle and the traffic types. It has been evaluated that the quality of service improves as we increases the speed of the vehicle from $36 \mathrm{~km} / \mathrm{hr}$ to $72 \mathrm{~km} / \mathrm{hr}$ because of vehicle with fast speed spends less time in dead zones (area which is outside the coverage of an access point) as compared to the vehicle with less speed thus the packet drop rate is less in case of $72 \mathrm{~km} / \mathrm{hr}$ as compared to $36 \mathrm{~km} / \mathrm{hr}$. If the performance comparison is done based upon the traffic type we found that STCP outperforms STG because of its additional applications like congestion detection and control services which are not present in STG. Thus for better performance in urban scenario the speed of the vehicle should be fast and the STCP traffic protocol should be preferred. This paper will be helpful to the active researchers which are working on different traffic types or in the field of communication networks.

\section{REFERENCES}

[1] Komal Sharma, Dr. Jyoteesh Malhotra, "A Comprehensive Survey on various Routing Protocols in VANET" International Journal of Scientific \& Technology Research, VOL 1, ISSUE 1 IJSTRC2014.

[2] Shie-Yuan Wang, NCTUns 5.0: A Network Simulator for IEEE 802.11(p) and 1609 Wireless Vehicular Network

Researches, Issue 1, Jan. 2013

[3] Francisco J. Martinez1, "A survey and comparative study of simulators for vehicular ad hoc network (VANETs) 2009 IEEE publications.

[4] Mostafa M. El-Said, "An Empirical Study To Compare Between IEEE 802.11p And Wave Protocols In Vanets Networks," Volume 4, Issue 4, July-August (2013), pp. 547-555. 


\section{ISSN 2277-3061}

[5] Shamsul Jamel Elias," A Comparative Study of IEEE 802.11 Standards for Non-Safety Applications on Vehicular Ad Hoc Networks: A Congestion Control Perspective ", Proceedings of the World Congress on Engineering and Computer Science 2014 Vol II WCECS 2014, 22-24 October, 2014, San Francisco, USA.

[6] Shivani Singh," Simulating VANET Scenario in Nctuns-6.0", National Workshop-Cum-Conference on Recent Trends in Mathematics and Computing (RTMC) 2011.

[7]Komal Sharma, Dr. Jyoteesh Malhotra "A Comprehensive Survey on Various Empirical Channel Models in VANET" Australian Journal of Information Technology and Communication Volume II Issue I, page 31-43,April-2015.

[8] Maria Fazio, Automatic IP Address Configuration in VANETs", Palazzi-Vanet2006.

[9] Saeed Gholamalitabar Firouzjaee, "Utilizing Mobile IP, MPLS to Improve QoS in VANET", Proc. of Int. Conf. on Advances in Signal Processing and Communication 2012.

[10] A GUI based user manual for NCTUns6.0 simulator. 